\begin{tabular}{|c|l|}
\hline Title & Residues of complex analytic foliations relative to singular invariant subvarieties \\
\hline Author(s) & Suwa, T. \\
\hline Citation & Hokkaido University Preprint Series in Mathematics, 254, 1-15 \\
\hline Issue Date & 1994-7-1 \\
\hline DOI & 10.14943/83401 \\
\hline Doc URL & http://hdl.handle.net/2115/69005 \\
\hline Type & bulletin (article) \\
\hline File Information & pre254.pdf \\
\hline
\end{tabular}

Instructions for use 
RESIDUES OF COMPLEX

ANALYTIC FOLIATIONS

RELATIVE TO SINGULAR

INVARIANT SUBVARIETIES

Tatsuo Suwa

Series $\sharp 254$. July 1994 


\section{HOKKAIDO UNIVERSITY}

\section{PREPRINT SERIES IN MATHEMATICS}

\#229: K. Tsutaya, Lower bounds for the life span of solutions of semilinear wave equations with data of non compact support, 14 pages. 1994.

$\sharp 230: \quad$ H. Okuda, I. Tsuda, A coupled chaotic system with different time scales: Toward the implication of observation with dynamical systems, 31 pages. 1994.

$\sharp 231$ : A. Hoshiga, The asymptotic behaviour of radial solutions near the blow-up point to quasi-linear wave equations in two space dimensions, 8 pages. 1994.

$\sharp$ 232: $\quad$ Y. Giga, N. Mizoguchi, Existence of periodically evolving convex curves moved by anisotropic curvature, 12 pages. 1994.

\#233: C. Dohmen, Y. Giga, N. Mizoguchi, Existence of selfsimilar shrinking curves for anisotropic curvature flow equations, 13 pages. 1994.

\# 234: T. Nakazi, M. Yamada, Invertible Toeplitz operators and uniform algebras, 14 pages. 1994.

\#235: C. Dohmen, A Pohozaev-type inequality for a quasilinear Haraux-Weissler equation, 6 pages. 1994.

$\sharp 236$ : T. Mikami, Large deviations for the first exit time on small random perturbations of dynamical systems, 32 pages. 1994.

$\sharp 237$ T. Ozawa, Characterization of Trudinger's inequality, 7 pages. 1994.

$\sharp 238$ T. Hibi, Buchsbaum complexes with linear resolutions, 12 pages. 1994.

\# 239 Y. Giga, N. Mizoguchi, On time periodic solutions of the Dirichlet problem for degenerate parabolic equations of nondivergence type, 23 pages. 1994.

$\sharp 240$ C. Dohmen, Existence of Fast Decaying Solutions to a Haraux-Weissler Equation With a Prescribed Number of Zeroes, 12 pages. 1994.

$\sharp 241$ K. Sugano, Note on H-separable Frobenius extensions, 8 pages. 1994.

\#242 J. Zhai, Some Estimates For The Blowing up Solutions of Semilinear Heat Equations, 11 pages. 1994.

$\sharp 243$ N. Hayashi, K. Kato and T. Ozawa, Dilation Method and Smoothing Effect of the Schrödinger Evolution Group, 10 pages. 1994.

$\sharp 244$ D. Lehmann, T. Suwa, Residues of holomorphic vector fields relative to singular invariant subvarieties, 26 pages. 1994.

\#245 H. Kubo, Slowly decaying solutions for semilinear wave equations in odd space dimensions, 30 pages. 1994.

$\sharp 246$ T. Nakazi, M. Yamada, ( $A_{2}$ )-Conditions and Carleson Inequalities, 27 pages. 1994.

$\sharp 247$ N. Hayashi, K. Kato and T. Ozawa, Dilation Method and smoothing Effect of Solutions to the Benjamin-ono Equation, 17 pages. 1994.

\# 248 H. Kikuchi, Sheaf cohomology theory for measurable spaces, 12 pages. 1994.

$\sharp 249$ A. Inoue, Tauberian theorems for Fourier cosine transforms, 9 pages. 1994.

$\sharp 250$ S. Izumiya, G. T. Kossioris, Singularities for viscosity solutions of Hamilton-Jacobi equations, 23 pages. 1994.

$\sharp 251$ H. Kubo, K. Kubota, Asymptotic behaviors of radially symmetric solutions of $\square u=|u|^{p}$ for super critical values $p$ in odd space dimensions, 51 pages. 1994.

\#252 T. Mikami, Large Deviations and Central Limit Theorems for Eyraud-Farlie-Gumbel-Morgenstern Processes, 9 pages. 1994.

$\sharp 253$ T. Nishimori, Some remarks in a qualitative theory of similarity pseudogroups, 19 pages. 1994 . 


\title{
RESIDUES OF COMPLEX ANALYTIC FOLIATIONS RELATIVE TO SINGULAR INVARIANT SUBVARIETIES*)
}

\author{
Tatsuo Suwa
}

In this article, we summarize the results in [LS] and, at the same time, supplement relevant material that are omitted there.

In section 1, we review the index and the index formula of C. Camacho and P. Sad [CS], which is the starting point of our research. If $S$ is a complex analytic surface and if $C$ is a compact non-singular curve in $S$, there is such a global invariant as the first Chern class $c_{1}\left(N_{C}\right)$ of the normal bundle $N_{C}$ of $C$ in $S$. It is determined by the way $C$ is embedded in $S$ and is equal to the self-intersection number $C^{2}$ of $C$ in this case. In [CS] it is found that, if there is a one dimensional complex analytic foliation (i.e., a foliation determined locally by a holomorphic vector field) on $S$ leaving $C$ invariant, at each of the points on $C$ which are singular points of the foliation, one can define an index and the sum of the indices is equal to $c_{1}\left(N_{C}\right)$. The formula was effectively used to prove the existence of a local integral curve (separatrix) for arbitrary holomorphic vector field with an isolated singularity at the origin in $\mathbb{C}^{2}$. In section 2 , after we review some basic definitions and facts about complex analytic singular foliations and their invariant subvarieties, we state the main results. Let $\mathcal{F}$ be an $s$-dimensional singular foliation on a complex manifold $W$ and $V$ a $p$-dimensional subvariety of $W$ which is left invariant by $\mathcal{F}$. We denote by $S(\mathcal{F}, V)$ the set of singular points of $V$ and singular points of $\mathcal{F}$ on $V$. We assume. that $V$ is a locally complete intersection and that its normal sheaf $N_{V}$, which is locally free on $V$, admits an extension $N$ (as a $C^{\infty}$ vector bundle) to a neighborhood of $V$. For each compact connected component $Z$ of $S(\mathcal{F}, V)$ and a symmetric and homogeneous polynomial $\varphi$ of degree $d>p-s$, we have the residue in the homology $H_{2 p-2 d}(Z ; \mathbb{C})$ of $Z$. Moreover, if $V$ is compact, then the sum of residues (taken in $H_{2 p-2 d}(V ; \mathbb{C})$ ) is equal to $\varphi(N) \cap[V]$ (Theorem (2.4)). In Theorem (2.7), we give an explict integral formula for the residue when $s=1$ and the singularity is isolated. In section 3 , we recall some basic material used to obtain these results. The key fact here is that the foliation induced on the invariant subvariety "acts" on the normal bundle of the subvariety away from the singularity. From this we have a Bott type vanishing theorem, which is the essential ingredient in defining the residues, i.e. in localizing the characteristic classes of $N_{V}$ to the singular set $S(\mathcal{F}, V)$. The actual

*) Report based on a joint work with D. Lehmann 
proofs and computations are conveniently done using the integration theory on the Cech-de Rham cohomology. The assumption that there is an extension $N$ is needed to construct forms to represent the characteristic classes of $N_{V}$ in the neighborhood of the singularity. These are explained in section 4 .

\section{The Camacho-Sad index}

We briefly recall the Camacho-Sad index of a holomorphic vector field on a complex surface relative to a (non-singular) invariant curve and their index theorem ([CS]). Let $X=A_{1} \frac{\partial}{\partial z_{1}}+A_{2} \frac{\partial}{\partial z_{2}}$ be a holomorphic vector field near the origin 0 in $\mathbb{C}^{2}=\left\{\left(z_{1}, z_{2}\right)\right\}$ with possibly an isolated singularity at 0 . Suppose that $X$ leaves the $z_{1}$-axis, denoted by $C$, invariant, which means that $X\left(z_{2}\right)$ is a multiple of $z_{2}$. Thus we may write $A_{2}=c z_{2}$, for some holomorphic function $c$ near 0 . Then the index of $X$ at 0 relative to $C$ is defined to be the residue of the meromoprphic 1-form $\left.\frac{c}{A_{1}}\right|_{z_{2}=0} d z_{1}$ on the $z_{1}$-plane. In fact it depends only on the foliation $\mathcal{F}$ generated by $X$ and not on $X$ itself. Thus we denote it by $\operatorname{Ind}_{0}(\mathcal{F}, C)$;

$$
\operatorname{Ind}_{0}(\mathcal{F}, C)=\frac{1}{2 \pi \sqrt{-1}} \int_{\gamma} \frac{c d z_{1}}{A_{1}}
$$

where $\gamma$ denotes a closed path in the $z_{1}$-plane $C$ going around 0 once counterclockwise.

(1.1) Example. If $X=\lambda_{1} z_{1} \frac{\partial}{\partial z_{1}}+\lambda_{2} z_{2} \frac{\partial}{\partial z_{2}}$ with $\lambda_{1}$ and $\lambda_{2}$ complex numbers, it leaves the $z_{1}$ axis invariant, and we compute

$$
\operatorname{Ind}_{0}(\mathcal{F}, C)=\frac{1}{2 \pi \sqrt{-1}} \int_{\gamma} \frac{\lambda_{2} d z_{1}}{\lambda_{1} z_{1}}=\frac{\lambda_{2}}{\lambda_{1}} .
$$

Note that $X$ also leaves the $z_{2}$-axis invariant and the index relative to that is $\frac{\lambda_{1}}{\lambda_{2}}$.

Thus the above index is not an integer, in general. Geometrically it is determined by the first order term in the holonomy.

Now let $S$ be a complex surface, i.e. a complex manifold of dimension 2 , and $C$ a compact non-singular curve in $S$. Suppose we have a one dimensional complex analytic foliation (foliation locally defined a single vector field) $\mathcal{F}$ on $S$ leaving $C$ invariant. Then the Camacho-Sad index theorem says that

$$
\sum_{p \in S(\mathcal{F})} \operatorname{Ind}_{p}(\mathcal{F}, C)=c_{1}\left(N_{C}\right)
$$

where $S(\mathcal{F})$ denotes the singular set of $\mathcal{F}$ and $c_{1}\left(N_{C}\right)$ the first Chern class of the normal bundle $N_{C}$ of $C$ in $S$, which is equal to the self-intersection number $C^{2}$ of C.

(1.2) Remarks. $1^{\circ}$. In the above formula, the right hand side is an integer which depends only on the way $C$ is embedded in $S$ and is independent of the foliation. 
$2^{\circ}$. The intersection number of two distinct curves is always localized to the points of intersection. On the other hand the self-intersection number of a curve may not be (complex analytically) localized. However, in the presence of a foliation leaving the curve invariant, it is localized to the singular points.

$3^{\circ}$. We are naturally led to consider generalizations in two directions. One is to consider the case of singular invariant curves. Generalizations in this direction are done by A. Lins Neto [LN1], M Soares [So] and the author [Su2]. It turns out that the natural global invariant is again the self-intersection number $C^{2}$ of the invariant curve $C$. The other is to consider the case of a higher dimensional singular foliation on a higher dimensional manifold leaving a (higher dimensional) submanifold invariant. This was worked out by J.-P. Brasselet (unpublished), B. Gmira [G] and Lins Neto [LN2] in special cases and by D. Lehmann [L3] in general. In the following sections we describe how it is generalized to the case of higher dimensional singular invariant subvarieties so that it includes the cases considered by the above authors.

\section{The residues}

For generalities on complex analytic singular foliations on complex manifolds, we refer to $[\mathrm{BB}]$ and [Sul]. Let $W$ be a (connected) complex manifold of dimension $n$ and $T W$ the holomorphic tangent bundle of $W$. We denote by $\mathcal{O}_{W}$ the sheaf of germs of holomorphic functions on $W$ and by $\Theta_{W}=\mathcal{O}_{W}(T W)$ the tangent sheaf on $W$. In general, for a coherent sheaf $\mathcal{S}$ on $W$, we set

$$
\operatorname{Sing}(\mathcal{S})=\left\{w \in W \mid \mathcal{S}_{x} \text { is not } \mathcal{O}_{W, w}-\text { free }\right\},
$$

where $\mathcal{S}_{w}$ and $\mathcal{O}_{W, w}$ denote the stalks at $w$ of the corresponding sheaves. The rank of $\mathcal{S}$ is defined to be the rank of the locally free sheaf $\left.\mathcal{S}\right|_{W-\operatorname{Sing}(\mathcal{S})}$.

We define (the tangent sheaf of) a singular foliation on $W$ to be an integrable coherent subsheaf of $\Theta_{W}$. Thus let $\mathcal{F}$ be a coherent subsheaf of $\Theta_{W}$. We set

$$
S(\mathcal{F})=\operatorname{Sing}\left(\Theta_{W} / \mathcal{F}\right)
$$

Note that $S(\mathcal{F})$ is an analytic set containing $\operatorname{Sing}(\mathcal{F})$. We may describe $S(\mathcal{F})$ explicitly as follows. Let $U$ be a sufficiently small coordinate neighborhood with complex analytic coordinates $\left(z_{1}, z_{2}, \ldots, z_{n}\right)$ and let $X_{1}, X_{2}, \ldots, X_{r}$ be generators of $\mathcal{F}$ on $U$. We write $X_{i}=\sum_{j=1}^{n} A_{i j}(z) \frac{\partial}{\partial z_{j}}$. Then we have

$$
S(\mathcal{F}) \cap U=\left\{z \in U \mid \operatorname{rank}\left(A_{i j}(z)\right)<s\right\},
$$

where $s$ is the rank of $\mathcal{F}$. 
(2.1) Definition. (The tangent sheaf of) a singular foliation on $W$ is a coherent subsheaf $\mathcal{F}$ of $\Theta_{W}$ which satisfies the "integrability condition", i.e.,

$$
\left[\mathcal{F}_{w}, \mathcal{F}_{w}\right] \subset \mathcal{F}_{w} \quad \text { for } w \in W-S(\mathcal{F}) .
$$

We say that $\mathcal{F}$ is reduced if it is full in $\Theta_{X}$, i.e., for any open set $U$ in $W$,

$$
\Gamma\left(U, \Theta_{X}\right) \cap \Gamma(U-S(\mathcal{F}), \mathcal{F})=\Gamma(U, \mathcal{F}),
$$

where $\Gamma(U ; \mathcal{S})$ denotes, for a sheaf $\mathcal{S}$, the space of sections of $\mathcal{S}$ on $U$.

(2.2) Remarks. $1^{\circ}$. If $s$ is the rank of $\mathcal{F}$, then $\mathcal{F}$ defines a non-singular foliation of dimension $s$ on $X-S(\mathcal{F})$. We call $\mathcal{F}$ a singular foliation of dimension $s$.

$2^{\circ}$. Given a singular foliation $\mathcal{F}$ on $W$. There is a canonical way of obtaining a reduced one from $\mathcal{F}$ ([Su1]).

$3^{\circ}$. If $\mathcal{F}$ is reduced, then the condition $\left({ }^{*}\right)$ holds for all $w$ in $W$.

$4^{\circ}$. If $\mathcal{F}$ is locally free, then $\mathcal{F}$ is reduced if and only if $\operatorname{codim} S(\mathcal{F}) \geq 2$.

Now let $V$ be a reduced and irreducible analytic subvariety of $W$ of dimension $p$. We denote by $\mathcal{I}_{V}$ the ideal sheaf of $\mathcal{O}_{W}$ defining $V$. Thus the quotient sheaf $\mathcal{O}_{V}=\mathcal{O}_{W} / \mathcal{I}_{V}$ is the sheaf of germs of holomorphic functions on $V$. We also denote by $\operatorname{Sing}(V)$ the singular set of $V$. We consider the sheaf $\Theta_{W}(\log V)$ of $\operatorname{logarithmic}$ vector fields of $V$ ([Sa], [BR]), i.e., $\Theta_{W}(\log V)=\left\{X \in \Theta_{W} \mid X\left(\mathcal{I}_{V}\right) \subset \mathcal{I}_{V}\right\}$. Note that a germ $X$ in $\Theta_{W}$ is in $\Theta_{W}(\log V)$ if and only if it is tangential to $V-\operatorname{Sing}(V)$. We define the tangent sheaf $\Theta_{V}$ of $V$ to be the images of the sheaf homomorphisms $\Theta_{W}(\log V) \otimes \mathcal{O}_{V} \rightarrow \Theta_{W} \otimes \mathcal{O}_{V}$. Note that there is a natural action of $\Theta_{V}$ on $\mathcal{O}_{V}$. The sheaf $\Theta_{V}$ is also defined as the dual of the sheaf of holomorphic 1-forms $\Omega_{V}$ on $V$, which is defined by the exact sequence

$$
\mathcal{I}_{V} / \mathcal{I}_{V}^{2} \rightarrow \Omega_{W} \otimes \mathcal{O}_{V} \rightarrow \Omega_{V} \rightarrow 0 .
$$

We have $\Theta_{V}=\operatorname{Hom}_{\mathcal{O}_{V}}\left(\Omega_{V}, \mathcal{O}_{V}\right)$ so that we have the exact sequence

$$
0 \rightarrow \Theta_{V} \rightarrow \Theta_{W} \otimes \mathcal{O}_{V} \stackrel{\pi}{\rightarrow} N_{V}
$$

where $N_{V}=\mathcal{H} m_{\mathcal{O}_{V}}\left(\mathcal{I}_{V} / \mathcal{I}_{V}^{2}, \mathcal{O}_{V}\right)$. The restriction of $N_{V}$ to $V-\operatorname{Sing}(V)$ is the sheaf of germs of holomorphic sections of the normal bundle of $V-\operatorname{Sing}(V)$ in $W$. Let $\mathcal{F}$ be a reduced $\operatorname{dim} s$ singular foliation and assume that

(1) $\mathcal{F}$ leaves $V$ invariant, i.e., $\mathcal{F} \subset \Theta_{W}(\log V)$, and that

(2) $V \not \subset S(\mathcal{F})$.

Thus $\mathcal{F}$ induces a $\operatorname{dim} s$ singular foliation on $V-\operatorname{Sing}(V)$, which may not be reduced. The singular foliation $\mathcal{F}_{V}$ on $V$ induced from $\mathcal{F}$ is defined to be the image of the sheaf homomorphism $\mathcal{F} \otimes \mathcal{O}_{V} \rightarrow \Theta_{W} \otimes \mathcal{O}_{V}$. Note that from the above condition 
(1) above, $\mathcal{F}_{V}$ is a subsheaf of $\Theta_{V}$. We set $S(\mathcal{F}, V)=\operatorname{Sing}(V) \cup(V \cap S(\mathcal{F}))$. In what follows, we assume that $V$ is a locally complete intersection of codimension $q=n-p$. Thus the sheaf $N_{V}$ is locally free on $V$. We further assume that there is a $C^{\infty}$ vector bundle $N$ on a neighborhood $U$ of $V$ which extend the holomorphic vector bundle, again denoted by $N_{V}$, defining the sheaf $N_{V}$. Note that every hypersurface satisfies the above assumptions. More generally, a subvariety defined by a regular section of a holomorphic vector bundle on $W$ satisfies the assumptions.

(2.4) Theorem. Let $W, \mathcal{F}$ and $V$ be as above and let $\varphi$ be a symmetric and homogeneous polynomial of degree $d>p-s$.

(1) If $Z$ is a connected component of $S(\mathcal{F}, V)$ which is compact, there is an element

$$
\operatorname{Res}_{\varphi}(\mathcal{F}, V ; Z) \in H_{2 p-2 d}(Z ; \mathbb{C})
$$

which depends only on the local behavior of $\mathcal{F}$ and $V$ near $Z$.

(2) If $V$ is compact, we have

$$
\sum_{Z} i_{*} \operatorname{Res}_{\varphi}(\mathcal{F}, V ; Z)=i^{*} \varphi(N) \cap[V] \quad \text { in } H_{2 p-2 d}(V ; \mathbb{C})
$$

where the first $i$ denotes the imbedding $Z \hookrightarrow V$ and the second $i$ the imbedding $V \hookrightarrow U$.

(2.5) Remarks. $1^{\circ}$. A symmetric polynomial $\varphi$ may be written as a polynomial $P\left(\sigma_{1}, \sigma_{2}, \ldots\right)$ of elementary symmetric functions $\sigma_{1}, \sigma_{2}, \ldots$ Then $\varphi(N)$ is defined to be the class in $H^{2 d}(U ; \mathbb{C})$ given by $\varphi(N)=P\left(c_{1}(N), c_{2}(N), \ldots\right)$.

$2^{\circ}$. If $V$ is non-singular, we have the Poincaré duality $H_{2 p-2 d}(V ; \mathbb{C}) \simeq H^{2 d}(V ; \mathbb{C})$ and the class $i^{*} \varphi(N) \cap[V]$ corresponds to $i^{*} \varphi(N)=\varphi\left(N_{V}\right)$. Thus the above formula reduces to the one in [L3].

$3^{\circ}$. If $V$ is a hypersurface in $W$, there is always a holomorphic line bundle $N$ on $W$ such that $V$ is defined by a regular section of $N$. Namely, if $\left\{U_{\alpha}\right\}_{\alpha \in A}$ is a covering of $W$ such that $V$ is defined by $f_{\alpha}=0$ on each $U_{\alpha}$, then $N$ is the line bundle on $W$ represented by the cocycle $\left\{f_{\alpha} / f_{\beta}\right\}$.

Next we give an explicit form of the residue in the case $s=1$ and $Z$ is an isolated point. Thus we consider the following situation. Let $X$ be a holomorphic vector field near the origin 0 in $\mathbb{C}^{n}$ and $V$ a complete intersection variety through 0 defined by reduced equations $f_{1}=\cdots=f_{q}=0$ with possibly isolated singularity at 0 . Assume that the set of zeros of $X$ intersects with $V$ only at 0 , thus $Z=\{0\}$.

(2.6) Lemma. There is a coordinate system $\left(z_{1}, \ldots, z_{n}\right)$ near the origin 0 in $\mathbb{C}^{n}$ such that if we write $X=\sum_{i=1}^{n} A_{i}(z) \frac{\partial}{\partial z_{i}}$, then $A_{1}, \ldots, A_{p}, f_{1}, \ldots, f_{q}, p=n-q$ form a regular sequence in $\mathcal{O}_{n}$.

For the proof of the above lemma, we refer to [LS]. 
From the condition that $V$ is invariant by $\mathcal{F}=(X)$, we may write $X\left(f_{i}\right)$ $=\sum_{j=1}^{r} c_{i j} f_{j}$ with $c_{i j}$ holomorphic functions near 0 . Let $C$ denote the matrix $\left(c_{i j}\right.$. For each $i=1,2, \ldots$, we define $\sigma_{i}(C)$ by

$$
\operatorname{det}(I+t C)=1+t \sigma_{1}(C)+t^{2} \sigma_{2}(C)+\cdots
$$

and for $\varphi=P\left(\sigma_{1}, \sigma_{2}, \ldots\right)$, we set $\varphi(C)=P\left(\sigma_{1}(C), \sigma_{2}(C), \ldots\right)$. In our case, since $s=1$, we have residues for polynomials $\varphi$ of degree $p=n-q$. Hence $\operatorname{Res}_{\varphi}(\mathcal{F}, V ;\{0\})$ is in $H_{0}(\{0\} ; \mathbb{C})=\mathbb{C}$ and is a number.

(2.7) Theorem. In terms of the coordinate system of Lemma (2.6), we have

$$
\operatorname{Res}_{\varphi}(\mathcal{F}, V ;\{0\})=\left(\frac{1}{2 \pi \sqrt{-1}}\right)^{p} \int_{\Gamma} \frac{\varphi(C) d z_{1} \wedge d z_{2} \wedge \cdots \wedge d z_{p}}{A_{1} A_{2} \ldots A_{p}}
$$

where $\Gamma$ is the p-cycle in $V$ defined by

$$
\Gamma=\left\{z|| A_{1}(z)|=\cdots=| A_{p}(z) \mid=\varepsilon, f_{1}(z)=\cdots=f_{q}(z)=0\right\}
$$

with orientation so that $d\left(\arg A_{1}\right) \wedge \cdots \wedge d\left(\arg A_{p}\right)$ is positive.

(2.8) Remark. In [LS], $\operatorname{Res}_{\varphi}(\mathcal{F}, V ;\{0\})$ is denoted by $I(\mathcal{F}, V, \varphi, \nu), \nu=N_{V}$ and $\varphi(C)$ already includes the constant $\left(\frac{\sqrt{-1}}{2 \pi}\right)^{p}$.

Now we consider some special cases of Theorem (2.7):

(I) The case $V$ is non-singular. In this case, it is obvious that the residue in Theorem (2.7) is identical with the one in [L3] Théorème 1. Especially if $p=q=1$ and $\varphi=\sigma_{1}$, we recover the index of [CS] (in section 1 , we denoted $\operatorname{Res}_{\sigma_{1}}(\mathcal{F}, C ;\{0\})$ by $\left.\operatorname{Ind}_{0}(\mathcal{F}, C)\right)$.

(II) The case $q=1$. In this case, since the rank of $N$ is one, for the polynomial $\varphi$, we have essentially $\varphi=\sigma_{1}^{p}, p=n-1$. Since $c_{1}(N)$ is the dual of the homology class $[V]$ in this case, the corresponding global invariant $i^{*} \varphi(N) \cap[V]$ in $H_{0}(V ; \mathbb{C})=\mathbb{C}$ is $c_{1}(N)^{n-1} \cap[V]=c_{1}(N)^{n}=V^{n}$, the self-intersection number of $V$ in $W$. If we write $X(f)=c f$, for some holomorphic function $c$ near 0 , we have

$$
\operatorname{Res}_{\varphi}(\mathcal{F}, V ;\{0\})=\left(\frac{1}{2 \pi \sqrt{-1}}\right)^{n-1} \int_{\Gamma} \frac{c^{n-1} d z_{1} \wedge d z_{2} \wedge \cdots \wedge d z_{n-1}}{A_{1} A_{2} \ldots A_{n-1}}
$$

where $\Gamma$ is the $(n-1)$-cycle in $V$ defined by

$$
\Gamma=\left\{z|| A_{1}(z)|=\cdots=| A_{n-1}(z) \mid=\varepsilon, f(z)=0\right\}
$$

with orientation so that $d\left(\arg A_{1}\right) \wedge \cdots \wedge d\left(\arg A_{n-1}\right)$ is positive. Especially, if $n=2$, we recover the index defined in [Su2] (which coincides with the one in [LN1], if $V$ is locally irreducible). 
(2.9) Example. Let $W=\mathbb{C}^{3}=\left\{\left(z_{1}, z_{2}, z_{3}\right)\right\}$ and $V$ the subvariety defined by

$$
f\left(z_{1}, z_{2}, z_{3}\right)=z_{1}^{\ell}+z_{2}^{\ell}+z_{3}^{\ell}=0 .
$$

If $X=\sum_{i=1}^{3} z_{i} \frac{\partial}{\partial z_{i}}$, then $X(f)=\ell f$. Since $z_{1}, z_{2}$ and $f$ form a regular sequence in this case, we have

$$
\operatorname{Res}_{\varphi}(\mathcal{F}, V ;\{0\})=\left(\frac{1}{2 \pi \sqrt{-1}}\right)^{2} \int_{\Gamma} \frac{\ell^{2} d z_{1} \wedge d z_{2}}{z_{1} z_{2}}
$$

where $\varphi=\sigma_{1}^{2}$ and $\Gamma$ is the 2-cycle in $V$ defined by

$$
\Gamma=\left\{z|| z_{1}|=| z_{2} \mid=\varepsilon, f(z)=0\right\}
$$

with orientation so that $d\left(\arg z_{1}\right) \wedge d\left(\arg z_{2}\right)$ is positive. On $\Gamma$, we have $z_{3}^{\ell}$ $=-\left(z_{1}^{\ell}+z_{2}^{\ell}\right)$. Thus if we set $z_{1}=\varepsilon e^{\sqrt{-1} \theta_{i}}, i=1,2, \Gamma$ is an $\ell$-fold covering of the set of $\left(\theta_{1}, \theta_{2}\right)$ in the square $[0,2 \pi]^{2}$. Since $\frac{d z_{1} \wedge d z_{2}}{z_{1} z_{2}}=-d \theta_{1} \wedge d \theta_{2}$, we get

$$
\int_{\Gamma} \frac{d z_{1} \wedge d z_{2}}{z_{1} z_{2}}=-4 \pi^{2} \ell
$$

Hence we have

$$
\operatorname{Res}_{\sigma_{1}^{2}}(F, V ;\{0\})=\ell^{3} \text {. }
$$

The natural compactification $V_{\ell}$ of $V$ in $\mathbb{C P}^{3}$ does not acquire singularities at infinity. The foliation $\mathcal{F}$ generated by $X$ can also extended to a foliation on $\mathbb{C P}^{3}$ which does not have singularities on $V_{\ell}$ at infinity ([LS]). Thus we recover:

$$
V_{\ell}^{3}=c_{1}^{2}(N) \cap\left[V_{\ell}\right]=\operatorname{Res}_{\sigma_{1}^{2}}(F, V ;\{0\})=\ell^{3}
$$

for the self-intersection number of $V_{\ell}$.

For more examples, see [LS].

\section{Relevant material}

(I) The Chern-Weil theory: For details, see, for example, $[\mathrm{B}],[\mathrm{BB}],[\mathrm{GH}]$ and [MS]. Let $W$ be a differentiable manifold (of class $C^{\infty}$ ) and $E$ a $C^{\infty}$ complex vector bundle of rank $r$. We denote by $C^{\infty}(W)$ the complex vector space of $C^{\infty}$ functions on $W$ and by $C^{\infty}(E)$ the $C^{\infty}(W)$-module of $C^{\infty}$ sections of $E$ on $W$. Denoting by $T_{\mathbb{R}} W^{-}$the real tangent bundle of $W$, we set

$$
\tau=T_{\mathbb{R}}^{c} W=T_{\mathbb{R}} W \otimes \mathbb{C} .
$$


Note that if $W$ is a complex manifold we have $\tau=T W \oplus \bar{T} W$. A connection $\omega$ for $E$ is determined by a $\mathbb{C}$-linear map

$$
\begin{gathered}
\nabla: C^{\infty}(E) \longrightarrow C^{\infty}\left(\tau^{*} \otimes E\right) \quad \text { with } \\
\nabla(f s)=d f \otimes s+f \nabla(s) \quad \text { for } f \in C^{\infty}(W) \text { and } s \in C^{\infty}(E) .
\end{gathered}
$$

For connections $\omega_{0}, \omega_{1}, \ldots, \omega_{k}$ on $E$ and for a symmetric and homogeneous polynomial $\varphi$ of degree $d$, we have a $2 d-k$ form $\Delta_{\omega_{0} \omega_{1} \cdots \omega_{k}}(\varphi)$ on $W$ such that

$$
d \Delta_{\omega_{0} \omega_{1} \cdots \omega_{k}}(\varphi)=\sum_{i=0}^{k}(-1)^{i} \Delta_{\omega_{0} \cdots \hat{\omega}_{i} \cdots \omega_{k}}(\varphi) .
$$

Thus, for a single connection $\omega, \Delta_{\omega}(\varphi)$ defines a class in the de Rham cohomology $H_{D R}^{2 d}(W ; \mathbb{C})$ depending only on $E$ and not on the connection chosen. We set

$$
\varphi(E)=\left(\frac{\sqrt{-1}}{2 \pi}\right)^{d}\left[\Delta_{\omega}(\varphi)\right] .
$$

Especially, when $\varphi=\sigma_{i}$, the $i$-th elementary symmetric polynomial, $\sigma_{i}(E)=c_{i}(E)$, the $i$-th Chern class of $E$.

(II) The vanishing theorem: Let $V$ be a $p$-dimensional (complex) submanifold of a complex manifold $W$ and $\mathcal{F}$ an $s$-dimensional foliation on $W$. Assume that $\mathcal{F}$ leaves $V$ invariant and that $S(\mathcal{F}) \cap V=\phi$. Thus $\mathcal{F}$ induces a non-singular $s$ dimensional foliation $\mathcal{F}_{V}$ on $V$. Note that $\pi$ in (2.3) is surjective in this case. The following lemma shows that there is a natural action of $\mathcal{F}_{V}$ on $N_{V}$ (Cf. [L3] Lemme Fondamental 2.1).

(3.1) Lemma. There is a $\mathbb{C}$-bilinear map

$$
\alpha: \mathcal{F}_{V} \times N_{V} \rightarrow N_{V}
$$

such that, if we denote $\alpha(\xi, \nu)$, for $\xi$ and $\nu$ in the stalks of $\mathcal{F}_{V}$ and $N_{V}$, respectively, over the same point, by $[\xi, \nu]$,

$$
[f \xi, \nu]=f[\xi, \nu] \text { and }[\xi, f \nu]=\xi(f) \nu+f[\xi, \nu] \quad \text { for } \quad f \in \mathcal{O}_{V}
$$

Proof. Denoting by 1 the germ in $\mathcal{O}_{V}$ represented by the function with the constant value 1 , a germ $\xi$ in $\mathcal{F}_{V}$ is written as $\xi=X \otimes 1$ (tensor product in $\Theta_{W} \otimes \mathcal{O}_{V}$ ) with $X$ in $\mathcal{F}$ and a germ $\nu$ in $N_{V}$ as $\pi(Y \otimes 1)$. If we set

$$
\alpha(\xi, \nu)=\pi([X, Y] \otimes 1)
$$


from the integrability of $\mathcal{F}$ and the fact that the sheaf homomorphism $\mathcal{F} \otimes \mathcal{O}_{V} \rightarrow$ $\Theta_{W} \otimes \mathcal{O}_{V}$ is injective away from $S(\mathcal{F})$, we see readily that it is well-defined. The rest of the lemma follows from the property of the Poisson bracket of vector fields.

On the complex manifold $V, \mathcal{F}_{V}$ and $N_{V}$ are locally free and $\mathcal{F}_{V}$ may be thought of as a subbundle of $T V$. We define

$$
\delta: C^{\infty}\left(N_{V}\right) \longrightarrow C^{\infty}\left(\left(\mathcal{F}_{V} \oplus \bar{T} V\right)^{*} \otimes N_{V}\right)=C^{\infty}\left(\operatorname{Hom}\left(\mathcal{F}_{V}, N_{V}\right) \oplus\left(\bar{T}^{*} V \otimes N_{V}\right)\right)
$$

by

$$
\delta(\nu)=(\xi \mapsto[\xi, \nu], \bar{\partial} \nu) .
$$

We call a connection $\omega$ for $N_{V}$ special if it is determined by $\nabla$ which extends $\delta$, i.e., if the following diagram is commutative:

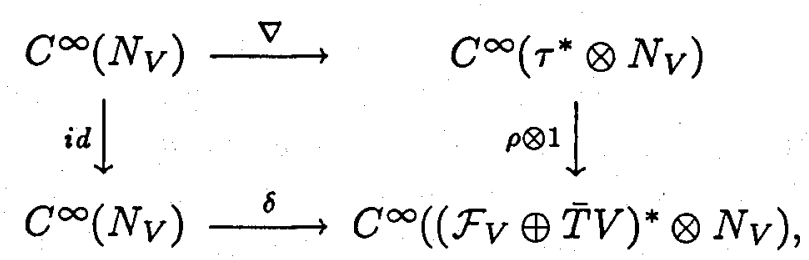

where $\rho$ denotes the canonical projection $\tau^{*} \rightarrow\left(\mathcal{F}_{V} \oplus \bar{T} V\right)^{*}$ (transposed of the natural inclusion $\left.\mathcal{F}_{V} \oplus \bar{T} V \rightarrow \tau\right)$. Note that, in general, a connection for a holomorphic vector bundle extends $\bar{\partial}$ if and only if the entries of the connection matrix with respect to a holomorphic frame are all of type $(1,0)$ ([BB] p.293). Note also that a special connection for $N_{V}$ always exists ([BB] Lemma (2.5)).

We quote the vanishing theorem ([L3] Théorème 2, [KT]), which can also be proved in a similar way as [BB] Proposition (3.27) and Lemma (5.3).

(3.2) Lemma. Let $\omega_{0}, \omega_{1}, \ldots, \omega_{k}$ be special connections for $N_{V}$. If $\varphi$ is a symmetric and homogeneous polynomial of degree $d>p-s$, then

$$
\Delta_{\omega_{0} \omega_{1} \cdots \omega_{k}}(\varphi)=0
$$

(III) Integration on the Cech-de Rham cohomology: For details on this subject, we refer to [BT], [L1] - [L3]. For an open set $U$ in a differentiable manifold $W$, we denote by $A^{q}(U)$ the space of (complex valued) differentiable $q$-forms on $U$. Let $\mathcal{U}=\left\{U_{i}\right\}_{i \in \mathcal{I}}$ be an open covering of $X$ whose index set $\mathcal{I}$ is a countable ordered set. We set

$$
\mathcal{I}^{p}=\left\{I=\left(i_{0}, \ldots, i_{p}\right) \mid i_{0}<\cdots<i_{p}, i_{\nu} \in \mathcal{I}\right\}
$$

and $\mathcal{I}^{*}=\bigcup_{p \geq 0} \mathcal{I}^{p}$. Also, for $I=\left(i_{0}, \ldots, i_{p}\right)$ in $\mathcal{I}^{p}$, we set $|I|=p$ and $U_{I}=U_{i_{0} \cdots i_{p}}$ $=\bigcap_{\nu=0}^{p} U_{i_{\nu}}$ and define $C^{p}\left(\mathcal{U}, A^{q}\right)$ to be the direct product

$$
C^{p}\left(\mathcal{U}, A^{q}\right)=\prod_{I \in \mathcal{I}^{p}} A^{q}\left(U_{I}\right) .
$$


Thus an element $\sigma$ in $C^{p}\left(\mathcal{U}, A^{q}\right)$ assigns to each $I$ in $\mathcal{I}^{p}$ an element $\sigma_{I}$ in $A^{q}\left(U_{I}\right)$. The coboundary operator $\delta: C^{p}\left(\mathcal{U}, A^{q}\right) \rightarrow C^{p+1}\left(\mathcal{U}, A^{q}\right)$ is defined by

$$
(\delta \sigma)_{i_{0} \cdots i_{p+1}}=\sum_{\nu=0}^{p+1}(-1)^{\nu} \sigma_{i_{0} \ldots i_{\nu} \cdots i_{p+1}}
$$

This together with the exterior derivative $d$ makes $C^{*}\left(\mathcal{U}, A^{*}\right)$ a doule complex. The (simple) complex obtained from this is denoted by $\left(A^{*}(\mathcal{U}), D\right)$. Thus $A^{r}(\mathcal{U})$ $=\bigoplus_{p+q=r} C^{p}\left(\mathcal{U}, A^{q}\right)$ and the differential $D: A^{r}(\mathcal{U}) \rightarrow A^{r+1}(\mathcal{U})$ is given by

$$
(D \sigma)_{I}=\sum_{\nu=0}^{p}(-1)^{\nu} \sigma_{I-i_{\nu}}+(-1)^{p} d \sigma_{I}
$$

for $I$ with $|I|=p$. We call $\left(A^{*}(\mathcal{U}), D\right)$ the Cech-de Rham complex and its cohomology $H^{*}\left(A^{*}(\mathcal{U})\right)$ the Čch-de Rham cohomology with respect to the covering $\mathcal{U}$. Since one of the spectral sequences associated to the double complex $C^{*}\left(\mathcal{U}, A^{*}\right)$ degenerates, we see that the restriction map $A^{r}(X) \rightarrow C^{0}\left(\mathcal{U}, A^{r}\right) \subset A^{r}(\mathcal{U})$ induces an isomorphism

$$
H^{r}(W ; \mathbb{C}) \simeq H^{r}\left(A^{*}(\mathcal{U})\right)
$$

of the de Rham cohomology onto the Cech-de Rham cohomology. The pairing

$$
A^{r}(\mathcal{U}) \times A^{s}(\mathcal{U}) \rightarrow A^{r+s}(\mathcal{U})
$$

defined, for $\sigma \in A^{r}(\mathcal{U})$ and $\tau \in A^{s}(\mathcal{U})$, by

$$
(\sigma \cup \tau)_{i_{0} \cdots i_{p}}=\sum_{\nu=0}^{p}(-1)^{(r-\nu)(p-\nu)} \sigma_{i_{0} \cdots i_{\nu}} \wedge \tau_{i_{\nu} \cdots i_{p}}
$$

induces the cup product

$$
H^{r}\left(A^{*}(\mathcal{U})\right) \times H^{s}\left(A^{*}(\mathcal{U})\right) \rightarrow H^{r+s}\left(A^{*}(\mathcal{U})\right)
$$

with the usual properties.

Next, we recall the integration theory on stratified spaces ([L1] - [L3]). Let $W$ be an oriented differentiable manifold of real dimension $N$ and $V$ a closed subset of $W$. Given a (locally finite) stratification $V=\coprod_{i \in \mathcal{I}^{\prime}} V_{i}$ of $V$ by submanifolds $V_{i}$ of $W$. Contrary to [L1], we order the index set $\mathcal{I}^{\prime}$ by $i \leq j$ if and only if $V_{j} \subset \bar{V}_{i}$. Let $U_{i}$ be a tubular neighborhood of $V_{i}$ in $W$. We set $U_{0}=W-V$ and $\mathcal{I}=\mathcal{I}^{\prime} \cup\{0\}$ and we put an order relation in $\mathcal{I}$ by $0<i$ for any $i$ in $\mathcal{I}^{\prime}$. We denote by $\mathcal{U}$ the 
covering $\left\{U_{i}\right\}_{i \in I}$ of $W$. We set $T_{0}=U_{0}$ and, for each $i$ in $\mathcal{I}^{\prime}$, we let $T_{i}$ be a closed disk bundle in $U_{i}$. Moreover, for each $i$ in $\mathcal{I}$, we set

$$
R_{i}=T_{i} \cap\left(\bigcap_{j>i}\left(X-\operatorname{Int} T_{j}\right)\right)
$$

and, for $I=\left(i_{0}, \ldots, i_{p}\right)$ in $\mathcal{I}^{p}$, we set $R_{I}=R_{i_{0}} \cap \cdots \cap R_{i_{p}}=\partial R_{i_{0}} \cap \cdots \cap \partial R_{i_{p}}$. We orient $R_{I}$ as in [L1]. If $W$ is compact, the integration

$$
\int_{W}: A^{N}(\mathcal{U}) \rightarrow \mathbb{C}
$$

defined by assigning to $\sigma$ in $A^{N}(\mathcal{U})$ the sum $\sum_{I \in I^{*}} \int_{R_{I}} \sigma_{I}$ induces integration on the cohomology $H^{N}\left(A^{*}(\mathcal{U})\right)$ which is compatible with the usual integration on the de Rham cohomology $H^{N}(W ; \mathbb{C})((3.3))$. Also the pairing

$$
B: A^{r}(\mathcal{U}) \times A^{N-r}(\mathcal{U}) \rightarrow A^{N}(\mathcal{U}) \rightarrow \mathbb{C}
$$

defined as the composition of the cup product and the integration induces the Poincaré duality

$$
H^{r}\left(A^{*}(\mathcal{U})\right) \simeq H_{N-r}(W ; \mathbb{C}) .
$$

Suppose now that $V$ is compact ( $W$ may not be). We denote by $A^{r}\left(\mathcal{U}, U_{0}\right)$ the kernel of the canonical projection $A^{r}(\mathcal{U}) \rightarrow A^{r}\left(U_{0}\right)$ and by $A^{r}\left(\mathcal{U}, \mathcal{U}^{\prime}\right)$ the kernel of the canonical projection $A^{r}(\mathcal{U}) \rightarrow A^{r}\left(\mathcal{U}^{\prime}\right)$, where $\mathcal{U}^{\prime}=\left\{U_{i}\right\}_{i \in \mathcal{I}^{\prime}}$, a covering of $V$. Note that the cohomology of the complex $A^{*}\left(\mathcal{U}, U_{0}\right)$ is $H^{*}(W, W-V ; \mathbb{C})$ and that of $A^{*}\left(\mathcal{U}^{\prime}\right)$ is $H^{*}(V ; \mathbb{C})$. Since the restriction of the cup product to $A^{r}\left(\mathcal{U}, U_{0}\right) \times$ $A^{N-r}\left(\mathcal{U}, \mathcal{U}^{\prime}\right)$ is zero, we have the paring

$$
B: A^{r}\left(\mathcal{U}, U_{0}\right) \times A^{N-r}\left(\mathcal{U}^{\prime}\right) \rightarrow \mathbb{C},
$$

which induces the Alexander-Lefschetz duality

$$
H^{r}(W, W-V ; \mathbb{C}) \simeq H_{N-r}(V ; \mathbb{C})
$$

\section{Proofs}

(I) Proof of Theorem (2.4): Let $S=\coprod_{\alpha} Z_{\alpha}$ be a decomposition of $S$ into connected components and assume that each $Z_{\alpha}$ is compact. For each $\alpha$, we take a stratification $\coprod_{i_{\alpha} \in \mathcal{I}_{\alpha}} V_{\alpha, i_{\alpha}}$ of $Z_{\alpha}$ by submanifolds $V_{\alpha, i_{\alpha}}$ of $W$. Also, let $U_{\alpha, i_{\alpha}}$ and $U_{1}$, respectively, be sufficiently small tubular neighborhoods of $V_{\alpha, i_{\alpha}}$ and of $V_{1}=V-S$. We denote by $\mathcal{U}_{\alpha}$ the covering $\left\{U_{\alpha, i_{\alpha}}\right\}_{i_{\alpha} \in \mathcal{I}_{\alpha}}$ of $U_{\alpha}=\bigcup_{i_{\alpha} \in \mathcal{I}_{\alpha}} U_{\alpha, i_{\alpha}}$ and by $\mathcal{U}$ the covering $\left\{U_{1}\right\} \cup\left(\bigcup_{\alpha} \mathcal{U}_{\alpha}\right)$ of $U=U_{1} \cup\left(\bigcup_{\alpha} U_{\alpha}\right)$. Note that we may assume that $U_{\alpha}$ and $U$ are regular neighborhoods of $Z_{\alpha}$ and of $V$, respectively. If 
we further set $\mathcal{I}=\{1\} \cup\left(\bigcup_{\alpha} \mathcal{I}_{\alpha}\right)$, then we have a decomposition $\mathcal{I}^{*}=\mathcal{I}_{1}^{*} \amalg\left(\amalg_{\alpha} \mathcal{I}_{\alpha}^{*}\right)$, where $\mathcal{I}_{1}^{*}$ denotes the set of sequences $I$ of indices in increasing order starting with 1. We take $R_{1} \subset U_{1}$ and $R_{\alpha, i_{\alpha}} \subset U_{\alpha, i_{\alpha}}$ as in section 3 and we use a variant of the integration theory described there. Thus if $V$ is compact, we have the evaluation

$$
\int_{V}: A^{2 p}(\mathcal{U}) \rightarrow \mathbb{C}
$$

which is defined by $\int_{V} \sigma=\sum_{I \in I^{*}} \int_{R_{I} \cap V} \sigma_{I}$ for $\sigma$ in $A^{2 p}(\mathcal{U})$. This defines a pairing

$$
B: A^{k}(\mathcal{U}) \times A^{2 p-k}(\mathcal{U}) \rightarrow \mathbb{C}
$$

defined by

$$
B(\sigma, \tau)=\int_{V} \sigma \cup \tau
$$

for $\sigma$ in $A^{k}(\mathcal{U})$ and $\tau$ in $A^{2 p-k}(\mathcal{U})$, which induces a map

$$
\kappa_{V}: H^{k}\left(A^{*}(\mathcal{U})\right) \simeq H^{k}(U ; \mathbb{C}) \rightarrow H_{2 p-k}(U ; \mathbb{C}) \simeq H_{2 p-k}(V ; \mathbb{C})
$$

which coinsides with the cap product with the homology class [ $V$ ]. If we take $\sigma$ with $\sigma_{1}=0$, then, from the definition (3.4), we see that the cup product $\sigma \cup \tau$ depends only on $\tau_{I}$ for $I$ in $\coprod_{\alpha} \mathcal{I}_{\alpha}^{*}$. So if we define $A^{k}\left(\mathcal{U}, U_{1}\right)$ by the exact sequence

$$
0 \rightarrow A^{k}\left(\mathcal{U}, U_{1}\right) \rightarrow A^{k}(\mathcal{U}) \rightarrow A^{k}\left(U_{1}\right) \rightarrow 0
$$

even if $V$ is not compact, we have the pairing

$$
B: A^{k}\left(\mathcal{U}, U_{1}\right) \times A^{2 p-k}\left(\bigcup_{\alpha} \mathcal{U}_{\alpha}\right) \rightarrow \mathbb{C}
$$

which induces a map

$$
\kappa_{V}: H^{k}\left(U, U_{1} ; \mathbb{C}\right) \rightarrow H_{2 p-k}\left(\bigcup_{\alpha} U_{\alpha} ; \mathbb{C}\right) \simeq H_{2 p-k}(S ; \mathbb{C})=\bigoplus_{\alpha} H_{2 p-k}\left(Z_{\alpha} ; \mathbb{C}\right)
$$

Now, if we denote by $\rho: U_{1} \rightarrow V_{1}$ the projection map, the restriction $\left.N\right|_{U_{1}}$ of $N$ to $U_{1}$ is diffeomorphic to the pull-back $\rho^{*} N_{V_{1}}$ of the normal bundle $N_{V_{1}}$ of $V_{1}$. Let $\omega$ be a special connection for $N_{V_{1}}$ and $\omega_{1}$ the connection for $N$ on $U_{1}$ defined by $\omega_{1}=\rho^{*} \omega$. On each $U_{\alpha, i_{\alpha}}$ we choose an arbitrary connection $\omega_{\alpha, i_{\alpha}}$ for $N$. The class $\varphi(N)$ in $H^{2 d}\left(A^{*}(\mathcal{U})\right)$ is then represented by a cocycle $\sigma$ with

$$
\sigma_{I}=\left(\frac{\sqrt{-1}}{2 \pi}\right)^{d} \Delta_{\omega_{I}}(\varphi)
$$


where $\Delta_{\omega_{I}}(\varphi)=\Delta_{\omega_{i_{1}} \cdots \omega_{i_{k}}}(\varphi)$ for $I=\left(i_{1}, \ldots, i_{k}\right)$. Since $\omega$ is special, we have $\Delta_{\omega_{1}}(\varphi)=0$. Hence, from the above remark, the class $\varphi(N)$ is in $H^{2 d}\left(A^{*}\left(\mathcal{U}, U_{0}\right)\right)$. We define the residue of $\mathcal{F}$ on $Z_{\alpha}$ relative to $V$ with respect to $\varphi$, denoted by $\operatorname{Res}_{\varphi}\left(\mathcal{F}, V ; Z_{\alpha}\right)$, to be the $\alpha$ component of $\kappa_{V}(\varphi(N))$ so that we may write

$$
\kappa_{V}(\varphi(N))=\sum_{\alpha} \operatorname{Res}_{\varphi}\left(\mathcal{F}, V ; Z_{\alpha}\right)
$$

It is now clear that we have the residue formula (2) above if $V$ is compact.

(4.1) Remarks. $1^{\circ}$. The above procedure can be summarized in the following diagram:

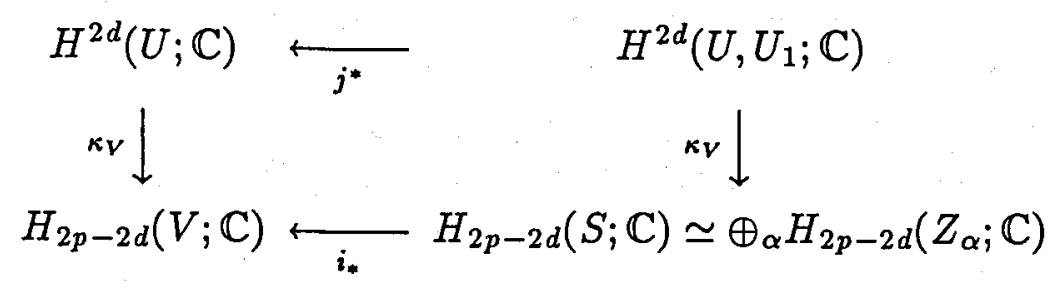

where $j^{*}$ denotes the canonical homomorphism. If we compute the class $\varphi(N)$ in $H^{2 d}(U ; \mathbb{C})$, which corresponds to $i^{*} \varphi(N) \cap[V]$ in $H_{2 p-2 d}(V ; \mathbb{C})$ by $\kappa_{V}$, using a special connection, we see that it has a natural reduction in $H^{2 d}\left(U, U_{1} ; \mathbb{C}\right)$ whose image by $\kappa_{V}$ is the sum $\sum_{\alpha} \operatorname{Res}_{\varphi}\left(E, V ; Z_{\alpha}\right)$ of the residues.

$2^{\circ}$. In fact, it is not necessary to take a stratification of $Z_{\alpha}$. Take a regular neighborhood $U_{\alpha}$ for each $Z_{\alpha}$ and work directly on the Čech-de Rham cohomology with respect to the covering $\left\{U_{1}\right\} \cup\left\{U_{\alpha}\right\}_{\alpha}$.

(II) Outline of the proof of Theorem (2.7): Let $U_{1}$ be a tubular neighborhood of $V_{1}=V-\{0\}$ with the projection $\rho: U_{1} \rightarrow V_{1}$ and $U_{\alpha}$ a small open disk about 0 . We consider the Čech-de Rham cohomology $H^{r}\left(A^{*}(\mathcal{U})\right)$ associated with the covering $\mathcal{U}=\left\{U_{1}, U_{\alpha}\right\}$ of $U=U_{1} \cup U_{\alpha}$. Here we recall the exact sequence of vector bundles

$$
\left.0 \rightarrow T V_{1} \rightarrow T U\right|_{V_{1}} \stackrel{\pi}{\rightarrow} N_{V_{1}} \rightarrow 0 .
$$

We denote by $\nu_{1}, \ldots, \nu_{q}$ the frame $\pi\left(\frac{\partial}{\partial f_{1}}\right), \ldots, \pi\left(\frac{\partial}{\partial f_{q}}\right)$ for the normal bundle $N_{V_{1}}$. Let $\omega_{1}$ be the connection for $N$ on $U_{1}$ given by $\omega_{1}=\rho^{*} \omega$, where $\omega$ is a special connection for $N_{V_{1}}$ on $V_{1}$. We take as $\omega_{\alpha}$ an arbitrary connection for $N$ on $U_{\alpha}$ for the moment. Since $\Delta_{\omega_{1}}(\varphi)=0$, by definition the residue is given by

$$
\operatorname{Res}_{\varphi}(\mathcal{F}, V ;\{0\})=\left(\frac{\sqrt{-1}}{2 \pi}\right)^{p}\left(\int_{R_{\alpha} \cap V} \Delta_{\omega_{\alpha}}(\varphi)+\int_{R_{1 \alpha} \cap V} \Delta_{\omega_{1} \omega_{\alpha}}(\varphi)\right),
$$

where $R_{\alpha}$ and $R_{1 \alpha}$ are defined as in section 3. In our case, denoting by $T_{1}$ a closed disk bundle over $V_{1}$ in $U_{1}$ and by $T_{\alpha}$ a closed disk in $U_{\alpha}$, we have $R_{1}$ $=T_{1} \cap\left(U-\operatorname{Int} T_{\alpha}\right)$ and $R_{\alpha}=T_{\alpha}$. Hence, $R_{1 \alpha}$ is the restriction of the bundle 
$\rho: T_{1} \rightarrow V_{1}$ to $L=\partial T_{\alpha} \cap V$, which is the link of the singularity of $V$ at 0 , and we have $R_{1 \alpha} \cap V=L$. In order to further reduce the computation, we take as $\omega_{\alpha}$ the connection for $N$ on $U_{\alpha}$ flat with respect to the frame for $N$ obtained by extending the frame $\rho^{*} \nu_{1}, \ldots, \rho^{*} \nu_{q}$ throughout $U_{\alpha}$. Then we have

$$
\operatorname{Res}_{\varphi}(E, V ;\{0\})=\left(\frac{\sqrt{-1}}{2 \pi}\right)^{p} \int_{L} \Delta_{\omega_{1} \omega_{\alpha}}(\varphi) .
$$

The rest of the proof is done in three steps:

1) Let $V_{i}=\left\{m \in L \mid A_{i}(m) \neq 0\right\}$ for $i=1, \ldots, p$ and consider the Cech-de Rham cohomology with respect to the covering $\mathcal{V}=\left\{V_{i}\right\}$ of $L$. We study the properties of the connection $\omega_{i}^{\prime}$ of $N$ on $V_{i}$ whose connection matrix with respect to the given trivialization is $C \frac{d z_{i}}{A_{i}}$.

2) Then we prove that $\Delta_{\omega_{1} \omega_{\alpha}}(\varphi)$, which is a cocycle on $L$, is cohomologous, when imbedded in the total Cech-de Rham complex $A^{*}(\mathcal{V})$, to the element $\tau$ in $A^{2 p-1}(\mathcal{V})$ defined by:

$$
\begin{cases}\tau_{I_{0}}=\Delta_{\omega_{1} \omega_{1}^{\prime} \omega_{2}^{\prime} \ldots \omega_{p}^{\prime}}(\varphi), & \text { for } I_{0}=(1, \ldots, p) \\ \tau_{I}=0, & \text { for any simplex } I \text { of } \operatorname{dim} \neq p-1 \text { in the nerve of } \mathcal{V}\end{cases}
$$

3) Finally, we prove that

$$
\tau_{I_{0}}=\frac{\varphi(C) d z_{1} \wedge d z_{2} \wedge \cdots \wedge d z_{p}}{A_{1} A_{2} \cdots A_{p}}
$$

Using integration on $A^{*}(\mathcal{U})$ as recalled in section 3 , this will achieve the proof. For details we refer to [LS].

\section{REFERENCES}

[BB] P. Baum and R. Bott, Singularities of holomorphic foliations, J. of Diff. Geom. 7 (1972), 279-342.

[B] R. Bott, Lectures on characteristic classes and foliations, Lectures on Algebraic and Differential Topology, Lecture Notes in Mathematics 279, Springer-Verlag, New York, Heidelberg, Berlin, 1972, pp. 1-94.

[BT] R. Bott and L.W. Tu, Differential Forms in Algebraic Topology, Graduate Texts in Mathematics 82, Springer-Verlag, New York, Heidelberg, Berlin, 1982.

[BR] J.W. Bruce and R.M. Roberts, Critical points of functions on analytic varieties, Topology 27 (1988), 57-90.

[CS] C. Camacho and P. Sad, Invariant varieties through singularities of holomorphic vector fields, Ann. of Math. 115 (1982), 579-595.

[G] B. Gmira, Une généralisation d'un théorème de C.Camacho et P.Sad relatif aux feuilletages holomorphes singuliers, Thèse de IIlème cycle, Lille, 1984. See also: Sur les feuilletages holomorphes singuliers de codimension 1, Publ. Matemàtiques 36 (1992), 229-240.

[GH] Ph. Griffiths and J. Harris, Principles of Algebraic Geometry, John Wiley \& Sons, New York, Chichester, Brisbane, Toronto, 1978. 
[KT] F.W. Kamber and Ph. Tondeur, Foliated Bundles and Characteristic Classes, Lecture Notes in Mathematics 493, Springer-Verlag, New York, Heidelberg, Berlin, 1975.

[L1] D. Lehmann, Intégration sur les variétés stratifiées, C. R. Acad. Sci. Paris 307, Sér. I (1988), 603-606.

[L2] - Variétés stratifiées $C^{\infty}$ : Intégration de Čech-de Rham et théorie de ChernWeil, Geometry and Topology of Submanifolds II, Proc. Conf., May 30-June 3, 1988, Avignon, France, World Scientific, Singapore, 1990, pp. 205-248.

[L3] _ Résidus des sous-variétés invariantes d'un feuilletage singulier, Ann. Inst. Fourier 41 (1991), 211-258.

[LS] and T. Suwa, Residues of holomorphic vector fields relative to singular invariant subvarieties, preprint.

[LN1] A. Lins Neto, Algebraic solutions of polynomial differential equatuins and foliations in dimension two, Holomorphic Dynamics, Mexico 1986, Lecture Notes in Mathematics 1345, Springer-Verlag, New York, Heidelberg, Berlin, 1988, pp. 192-232.

[LN2] Complex codimension one foliations leaving a compact submanifold invariant, Dynamical Systems and Bifurcation Theory 1985, Pitman Research Notes in Mathematics Series 160, Longman Scientific and Technical, Harlow, New York, 1987, pp. 295-317.

[MS] J.W. Milnor and J.D. Stasheff, Characteristic Classes, Annales of Mathematics Studies 76, Princeton University Press, Princeton, 1974.

[Sa] K. Saito, Theory of logarithmic differential forms and logarithmic vector fields, J. Fac. Sci. Univ. Tokyo Sect. 1A Math. 27 (1980), 265-291.

[So] M. Soares, $A$ note on algebraic solutions of foliations in dimension 2, Dynamical Systems 1990, Pitman Research Notes in Mathematics Series 285, Longman Scientific and Technical, Harlow, New York, 1993, pp. 250-254.

[Su1] T. Suwa, Unfoldings of complex analytic foliations with singularities, Japanese J. of Math. 9 (1983), 181-206.

[Su2] _ Indices of holomorphic vector fields relative to invariant curves, preprint.

Department of Mathematics, Hokkaido University, Sapporo 060, Japan

E-mail address: suwa@math.hokudai.ac.jp 